\title{
Chronic low back pain and sick-leave: a functional magnetic resonance study
}

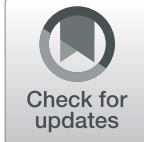

\author{
Aloma A. Feitosa', Edson Amaro Junior², Liana Guerra Sanches², Eduardo Ferreira Borba', \\ Liliana Lourenço Jorge ${ }^{2}$ and Ari Stiel Radu Halpern ${ }^{1,2^{*}}$ (i)
}

\begin{abstract}
Background: Chronic low back pain (CLBP) represents a problem in the occupational environment, often associated with disability, sick-leave demands, loss of productivity, anxiety, depression and high socioeconomic cost. The emergence of functional neuroimaging allowed new insights into brain structure and physiology in normality and chronic pain. While occupational related aspects are recognized as important risk factors for chronicity there have not been thus far evaluated by fMRI experiments. The overall objective of this study is to compare the neuronal correlates between groups of individuals CLBP with or without sick-leave demands.
\end{abstract}

Methods: A total of 74 individuals were divided into three groups: chronic low back pain with sick-leave demands [CLBP_L]; chronic low back pain without sick-leave demands [CLBP_NL]; individuals without pain or sick-leave demands [Control]. Functional magnetic resonance imaging was used to assess brain function during moderate acute pain stimulation task (thumb controlled pressure).

Results: After acute painful stimulation, a higher brain response was found in the anterior cingulate and superior and medium frontal gyrus was observed in CLBP_NL vs. CLBP_L $(p<0,001)$ and increased brain response in the frontal pole and paracingulate region in control vs. CLBP_L $(p<0.001)$ during acute pain stimulation.

Conclusion: The modulation of acute pain participates in the mechanism propagating chronic pain perception. The lower activation in the superior frontal gyrus observed in the CLBP_L group compared to CLBP_NL, reinforces the idea of an already existing activation in this area.

Keywords: Musculoskeletal, Sick-leave demands, Back disorders, Functional neuroimaging, Neuronal plasticity

\section{Background}

Low back pain (LBP) is one of the most common musculoskeletal complaints in industrialized societies, affecting $80 \%$ of adults at some time during their lives. Most pain episodes are self-limiting, but relapses are frequent and about $10 \%$ of individuals develop chronic pain $[1,2]$. LBP is a symptom that may be related to several alterations involving structures of the spine itself or not. However, often no correlation is found between clinical

\footnotetext{
* Correspondence: ari@arthron.com.br

${ }^{1}$ Rheumatology Division, Hospital das Clinicas HCFMUSP, Faculdade de Medicina, Universidade de São Paulo, Av. Dr. Arnaldo, n 455, Sao Paulo, SP, Brazil

${ }^{2}$ Instituto do Cérebro - Hospital Israelita Albert Einstein, Av Albert Einstein $627 / 701$ BA, 2SS, Sao Paulo, SP, Brazil
}

radiological assessments and low back pain symptoms. This is true for most cases of chronic low back pain, corresponding to a clinical entity named "common mechanical low back pain" or "nonspecific low back pain" [3].

Previous authors have reinforced the role of psychosocial factors in the evolution from acute to chronic LBP - CLBP [4, 5]. The poor physiologic understanding of CLBP is reflected in the characteristic inconstant and high variable response to treatment and also high recurrence rate $[4,6]$. Pain is a complex and subjective experience encompassing the interpretation of nociceptive stimuli influenced by memories, emotions, pathological, genetic and cognitive factors. It is associated with working environment mis adjustments, acquired movement disability, sick-leave demands, loss of productivity, 
anxiety, depression and high socioeconomic cost $[6,7]$. Since Waddel's [8], the correlation between LBP and sick-leave demands has been strongly emphasized. Despite all prevention efforts, the prevalence of disability due to LBP has increased considerably over the last decades in Western countries. In Brazil the picture is quite similar, CLBP was the first cause of sickness payment and the third cause of disability-related retirement [9, 10]. According to Brazilian Social Security data, back pain is the most frequent disease leading to sick-leave demands beyond 15 days in the past years (http://www. brasil.gov.br/economia-e-emprego).

Chronic low back pain (CLBP) is well known chapter in the occupational health context. Sick-leave demands is assured by the National Institute of Social Security (INSS) to the Brazilian worker provided the symptoms are adequately evaluated, and the disease is diagnosed by a certified doctor. In this context, pain is quite an obscure area since subjects complains are difficult to ascertain if no physical evidence is found. Even more, is the person has interests in obtaining benefits from sick-leave demands, it is often very difficult, if not impossible and even for experts - to disentangle possible bias without a robust measure of pain relevance other than selfreported evaluation.

The emergence of new techniques of functional neuroimaging allowed a great advance in the research and understanding about structure and brain functioning in normality and in several pathological conditions including CLBP [11, 12]. Functional Magnetic Resonance Imaging (fMRI) provides an indirect measurement of brain neuronal activity. The most often used fMRI measure is and endogenous contrast based on blood oxygenation - the BOLD (Blood Oxygenation Level Dependent) effect. It infers neural activity from localized changes in brain blood flow and rate of oxy/deoxyhaemoglobin changes in response to neural metabolic demand [13, 14]. However, the technique is mostly based on alternating moments.

Transient painful stimuli activate a large set of coordinated brain regions regardless of the modality used to provoke pain [15]. These regions collectively referred as the pain matrix has been extensively studied. It includes areas involved in sensory-discriminative aspects of pain (primary and secondary somatosensory cortex, thalamus and the posterior part of the insula) as well as areas implicated in emotional or affective aspects (anterior cingulate cortex, anterior part of the insula, prefrontal cortex) $[11,12]$. There is a growing evidence for an altered neural plasticity in chronic pain and specific chronic pain conditions are associated with distinct brain responses to acute pain, although sharing a few substrates [16]. Apkarian et al. [12], related that anatomy and physiology of the brain in chronic pain is distinct from that of healthy subjects experiencing acute pain. CLBP individuals are more sensitive and activate a greater number of cortical areas related to the pain matrix both with thermal or pressure stimuli $[17,18]$. Although functional imaging has provided important new insights into chronic pain conditions, the identification of subgroups of individuals with nonspecific CLBP remains a challenge. While occupational related aspects are recognized as important risk factors for chronicity there have not been thus far evaluated by fMRI experiments. We are not aware of any study using fMRI comparing CLBP with and without sick-leave demands and asymptomatic control subjects.

In this paper, we compared brain hemodynamic response from individuals CLBP with sick-leave demands to subjects with CLBP, but without sick-leave demands when submitted to acute pain matched by individual intensity perception and controlled by objective pressure. Our hypothesis is that subjects with CLBP with sick-leave demands would have a distinct pattern of brain activation influenced by the relationship between pain autoperception levels and objective pressure measurements.

\section{Materials and methods Subjects}

The study was carried out at the Hospital Israelita Albert Einstein (HIAE). Individuals were recruited from the outpatient of Rheumatology Division of Hospital das Clinicas HCFMUSP, Faculdade de Medicina, Universidade de Sao Paulo and Department of Physical Therapy of HIAE and through announcements made at these two institutions during the year of 2015. Initially 237 individuals were contacted by telephone and by email. A total of 135 did not meet the criteria for the study and were excluded. A further 24 individuals were excluded after a medical evaluation performed at the rheumatology outpatient unit of the HCFMUSP. Seventy-eight individuals were selected for the study. Four individuals did not tolerate the fMRI procedure and withdrew from the study. Seventy-four individuals, right-handed (44 women and 30 men) completed the study. All individuals signed the informed consent form. The study was approved by the Ethics Committee of both institutions (CAPPesq 0186/ 11 e CEP/Einstein $n^{\circ} 10 / 1480$ ).

We selected three groups of subjects:

1- individuals with CLBP and sick-leave demands (CLBP_L);

2- individuals with CLBP without sick-leave demands (CLBP_NL);

3- individuals without any form of chronic pain or sick-leave demands (Control).

The three-group design was used to promote a stricter comparison regarding the mechanisms related to sick- 
leave demands and not CLBP (CLBP_NL) and a baseline comparison to normal expected brain response to the same stimulus in subjects without a CBPL component (Control).

\section{Inclusion criteria for CBLP groups}

1-) localized pain between the last ribs and the gluteal folds, present most of the time for more than 12 weeks

2-) Numeric Rating Scale (NRS) pain score in the last 7 days $\geq 5$ (ranging from 0 to 10 )

3-) Functional disability defined by a score $\geq 10$ in the Rolland Morris questionnaire [19]

4-) Age between 35 and 60 years

5-) Right-handed individuals

\section{Inclusion criteria for the CLBP_L group}

Sick-leave demands was verified by an initial interview. Workers with back pain were included if on temporary sick-leave demands or requiring sick-leave demands.

\section{Inclusion criteria for control group}

No previous history of CBLP as investigated in a clinical interview by a trained rheumatologist.

\section{Exclusion criteria for all groups}

Diagnosis of inflammatory joint disease; Lumbar pain with radiation to the lower limbs below the knees or with characteristic nerve root dermatomal distribution; Diagnosis of spinal stenosis; History of spine surgery; Diagnosis of symptomatic lower limb osteoarthritis; Current or previous vertebral fractures; Current or previous diagnosis of neoplasia; Decompensated underlying chronic disease that, at the medical judgment, would impair the analysis; Subjects illiterate or unable to understand instructions.

\section{Experimental pain assessment}

Two devices were developed (Zurc \& Zurc Ltda, São Paulo, Brazil) based on a similar apparatus used by Gracely et.al [20]. intended to accurately synchronize pressure painful stimuli with image MRI acquisition. One device was operated manually and was used to evaluate the pain threshold before image acquisition, outside the MR scanning room (A1). It is composed of a metallic platform where weights are placed for calibration, providing stimulus pressure by a hydraulic circuit.

The second device was designed to operate inside the MR scanning room with materials based in acrylic and other non-ferromagnetic materials (A2). It operates in a similar way; however, it is activated by a computercontrolled pneumatic system. In both devices, the individual holds a stick for correct positioning of the left thumb inside a box in which a plunger exerts a set pressure to the nail surface for each experiment. Custom software synchronizes delivery of painful stimuli and fMRI image acquisition.

\section{Procedures on the day of data collection Self-assessment questionnaires}

Subjects answered the following previously validated questionnaires:

- Questionnaire of socio-economic classification (ABA-ABIPEME Criterion of the Brazilian Association of Advertisers - Brazilian Association of Market Research Institutes, Brazil 2008).

- Beck Depression Inventory (BDI) composed of 21 items with scores ranging from 0 to 63 . Scores above 15 denotes depressive symptoms [21].

- Anxiety Inventory (IDATE) which is divided into two questionnaires evaluating anxiety as a state (IDATE-E) and anxiety as a trait (IDATE-T). The anxiety state reflects a transient reaction directly related to a situation of adversity whereas the anxiety trait refers to a more stable aspect related to the propensity of the individual throughout life. The score varies from 20 to 80 points, and higher values indicate higher levels of anxiety [22].

- Brazilian version of the Fear Avoidance Questionnaire (FABQ) composed of two subscales FABQ-Phys (ranging from 0 to 24) and FABQ Work (ranging from 0 to 42). Higher scores indicate greater Fear-Avoidance [23].

\section{Evaluation of the pain threshold}

The subjects were sit on a chair next to the apparatus used to assess the pain threshold, this equipment was protected by a screen so the subjects could not see the weight used for adjusting the force applied to the finger nail. Subject hand only to sustain the device where he or she would receive the pressure stimuli. The pain sensitivity to pressure was verified through a threshold of supra-threshold sensations, descriptor of pain intensity and discomfort, this evaluation was performed before the acquisition of the images, using numerical pain scale (NRS) ranging from 0 to 10.

The subjects were submitted to increasing pressor stimulation to the left thumb, with duration of $5 \mathrm{~s}$, with the aid of rigid rubber probe, connected to a piston that operated manually, apparatus mentioned above (A1). The stimuli were applied to grade the intensity and discomfort of the painful sensation evoked, beginning with $1 \mathrm{~kg}-\mathrm{cm} 2$ and gradual increase of $0.5 \mathrm{~kg}-\mathrm{cm} 2$ until the individual reported a pressure capable of producing a medium-intensity pain ( 7 points in NRS), or up to a maximum of $7 \mathrm{~kg} / \mathrm{cm} 2$. The subject received a 
maximum of 13 stimuli (with intensity of $1 ; 1.5 ; 2 ; 2.5 ; 3$; $3.5 ; 4 ; 4.5 ; 5 ; 5.5 ; 6 ; 6.5 ; 7 \mathrm{~kg} / \mathrm{cm} 2$ ) applied only once. The weight used to induce medium intensity pain was increased by $1 \mathrm{~kg} / \mathrm{cm} 2$ and later used in the pain paradigm performed within the functional magnetic resonance imaging apparatus at the time of data collection.

A pilot study showed that subjects undergoing repeated pain stimulated habituation to pain and reduced pain intensity at the end of the study compared to the initial test. Adding $1 \mathrm{~kg}$ to the predetermined value. This step ensured delivery of the supra-threshold pain-stimulus.

\section{Data collection - fMRI acquisition}

Individuals were positioned in the MR system in dorsal decubitus position. The pressure device was placed on the left thumb. Total MR experiment lasted approximately $50 \mathrm{~min}$, including anatomical image acquisition and paradigm pain. All images were acquired in 3.0 Tesla MR System (Siemens Tim Trio, Erlangen, Germany) with 12 channel head coil. The visual stimuli presentation and subject response synchronization were made using eye goggles and a trigger box from NNL Systems (Nordic Neuro Lab, Bergen, Norway). The fMRI acquisition was based on $\mathrm{T} 2 \%$-weighted echo planar (EPI) images for the whole brain. Thirty two axial slices were acquired following AC-PC angulation. The acquisitions parameters for fMRI were: $\mathrm{TR}=2000 \mathrm{~ms}$, $\mathrm{TE}=30$ $\mathrm{ms}, 3,2 \mathrm{~mm}$ of slice thickness, $0.8 \mathrm{~mm}$ of interslice gap, FOV $=210 \mathrm{~mm}$, matrix 64X64 (isotropic voxels), discarding the first 4 volumes, necessary for the MR signal to reach steady state. We used an event-related fMRI paradigm. Pain (pressure) was maintained constant for $2 \mathrm{~s}$, with $14 \mathrm{~s}$ of rest. The Interstimulus interval was fixed (16s). The acquisition duration of 180 volumes was 5:06 min. We also collected T1-weighted sagittal highresolution structural image with 192 slices for coregistration with the fMRI data with the following. Parameters: T1-weighted GRE (MPRAGE) $1 \mathrm{~mm}$ isotropic voxel, $\mathrm{TR}=2500 \mathrm{~ms}, \mathrm{TE}=3.45 \mathrm{~ms}, \mathrm{FOV}=265 \mathrm{~mm}, \mathrm{IR}=$ 1100, flip angle 7 degrees.

\section{Image processing}

Data processing and statistical analyses were conducted using FSL (www.fmrib.ox.ac.uk/fsl/). The volumes were processed by movement correction (MCFLIRT), high pass filter cutoff (50s), spatial smoothing $(\mathrm{FWHM}=5$ $\mathrm{mm}$ ) and spatial normalization to standard space (affine, $12 \mathrm{DoF}$ ). The activation maps were produced using FILM routines for the general linear model (GLM), which is based on semiparametric estimation of residuals autocorrelation [24]. Statistical images were thresholded using Gaussian random field-based cluster inference with a threshold of $\mathrm{Z}>2.3$ at the voxel level and a corrected cluster significance threshold of $P<0.05$ FDR (False Discovery Rate) corrected.

A covariance analysis was also performed considering the pressure applied during the pain task as covariate for BOLD effect.

fMRI behavioural data was analyzed using KruskalWallis tests to compare the three groups and MannWhitney tests for two groups. Results are presented for estimated odds ratios, 95\% confidence intervals and $p$ values. The significance level adopted was $5 \%$ and the analyses were performed with the aid of the R package [25-27].

\section{Results}

Seventy-four individuals were included and were divided into three groups: CLBP_L $(N=24)$, CLBP_NL $(n=25)$ and controls $(n=25)$. No significant differences were observed in mean age $(48.5 \pm 5.7$ vs. $48 \pm 6$ vs. $45 \pm 7$ years, respectively, $p=0.530$ ) and frequencies of female gender ( $68 \%$ vs. $68 \%$ vs. $41.7 \%$, respectively, $p=0.097$ ).

The groups were homogeneous regarding body mass index (BMI) and socioeconomic classification. The two groups of CLBP individuals showed no difference in NRS.

In the CLBP_L, no patient was on definitive sick-leave demands; 10 patients (42\%) were away for more than 5 years.

Table 1 shows that CLBP_L were significantly more depressive ( $p=0.030$ vs control), had lower education $(p=0.010$ vs CLBP_NL; $p=0.048$ vs control), had greater functional disability measured by QRM $(p=$ 0.003 vs CLBP_NL), presented higher scores for IDATE$\mathrm{T}(p=0.004$ vs CLBP_NL; $p=0.033$ vs control $)$ and IDATE-E $(p<0.001$ vs CLBP_NL; $p<0.001$ vs control), presented higher FABQ-work ( $p=0.001$ vs CLBP_NL) but not in the FABQ-physic. All FABQ-work questions were significantly more frequent in the CLBP_L group. The mean pressure used to provoke pain of moderate intensity was lower for CLBP_NL ( $p<0.001$ vs controls).

\section{fMRI results - pain task}

All study groups showed acute pressure pain evoked activation in areas related to the pain matrix: S1, S2, thalamus, insula, prefrontal cortex and anterior cingulate cortex, without significant differences between groups (Fig. 1). When we performed the covariate analysis considering the weight used in the pain test, because the groups needed different weight to produce the same intention of moderate pain, verified significant differences in comparison CLBP_NL > CLBP_L with higher intensity voxels on the anterior cingulate and upper and middle frontal gyrus $(p<0.001)$ and in the comparing the group Controls > CLBP_L, we found significant differences in the frontal pole and paracingulate region $(p<0.001)$ (Fig. 2). No statistical difference was observed when other covariates were tested. 
Table 1 Group characteristics and results of pressure pain tests

\begin{tabular}{|c|c|c|c|c|}
\hline Variables & $C_{C B P} \_\mathrm{L}(n=24)$ & CLBP_NL $(n=25)$ & Control $(n=25)$ & $P$ value \\
\hline BMI (Kg / m2) & $27.5(25.1-29.6)$ & $26.3(24.8-29.5)$ & $25.8(24.6-29)$ & 0.678 \\
\hline Education, (years) & $10.5(8-11) * \#$ & $11(11-15)$ & $11(9-15)$ & 0.025 \\
\hline Disability (QRM) & $18(17-19.8)$ & $14(11-16.8)$ & NA & 0.003 \\
\hline NRS & $8(8-8.75)$ & $8(6.5-8.7)$ & NA & 0.709 \\
\hline depressive symptoms - N (\%) & $12(50) \#$ & $8(33.3)$ & $3(12)$ & 0.016 \\
\hline IDATE-T & $50.5(41.5-55.5)^{*} \#$ & $42(35.7-52)$ & $34(30-43)$ & $<0.001$ \\
\hline IDATE-E & $45(37.7-51)) * \#$ & $39(35.5-42)$ & $38(30-45)$ & 0.019 \\
\hline FABQ-physic & $18(10-22.2)$ & $10.5(3-17.2)$ & NA & 0.056 \\
\hline FABQ-work & $33,5(26.8-39.8)$ & $15,5(5.2-31)$ & NA & 0.001 \\
\hline Pressure (Kg) & $5(4.5-7.5)$ & $5(4.5-6) \#$ & $7(5.5-7.5)$ & 0.008 \\
\hline
\end{tabular}

Values were expressed by median and interquartile range (1st and 3rd quartiles) ${ }^{*} P<0.05$ vs. CLBP_NL; \# $P<0.05$ vs Control / CLBP_NL: individuals with low back pain and sick leave / CLBP_L: individuals with low back pain / $\mathbf{P}$ value: Hypothesis test of difference between the three groups and their categories / BMI: body mass index / Pressure: weight in Kg used to check pain threshold

\section{Discussion}

Chronic low back pain has been recognized as a Western epidemic for a several decades and the prevalence of CLBP is still increasing. The reason why a significant minority of low back pain individuals evolves into a chronic pain state remains unclear. Current theory supports the role of biological, psychological, and environmental factors in the etiology, exacerbation, and maintenance of chronic pain. These factors impact the occupational setting such that low back pain often leads to long-term

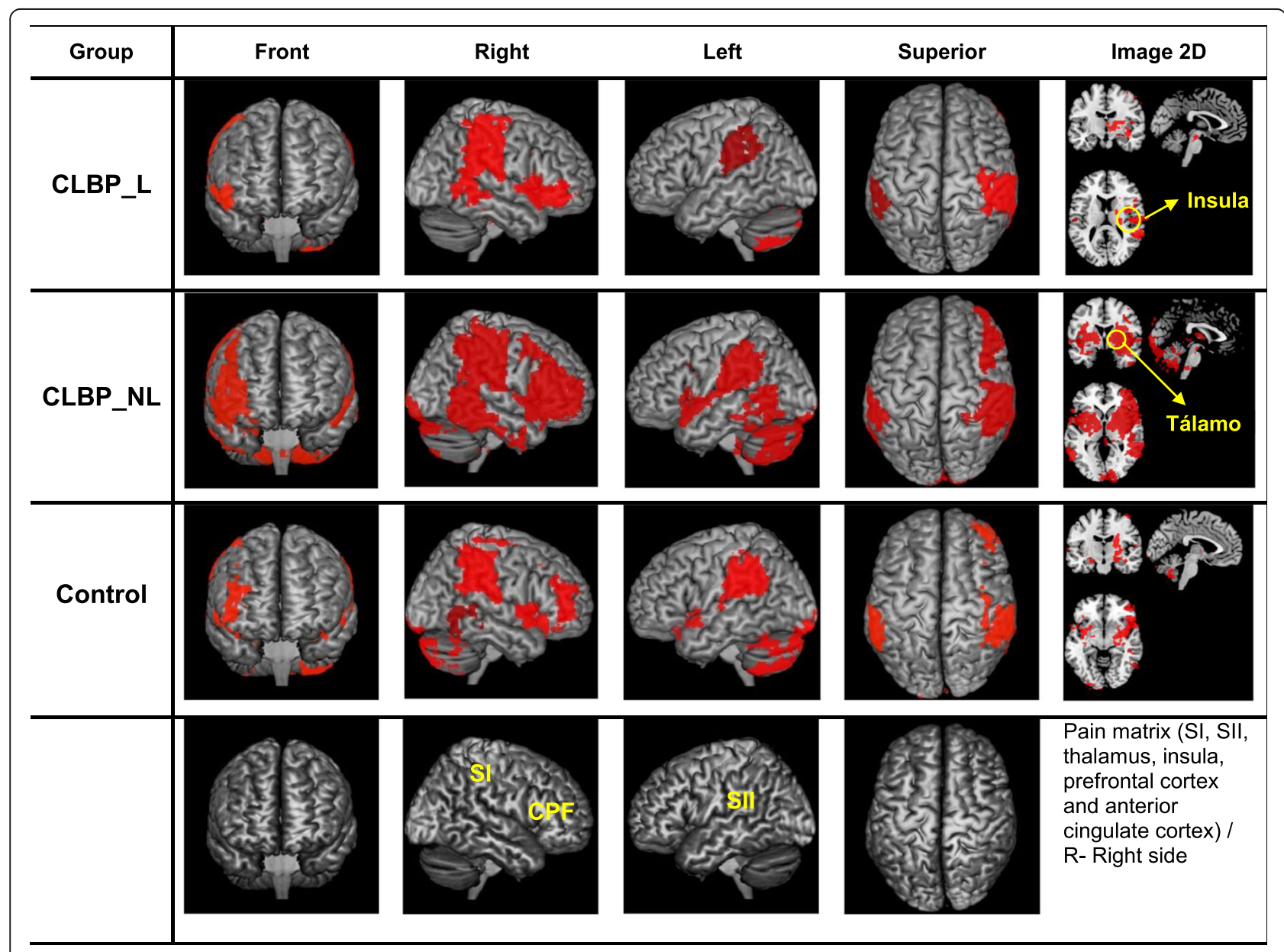

Fig. 1 Hemodynamic response, after painful stimulation, in the three groups studied, showed activation in the areas related to the pain matrix 
Result of fMRI analysis in pain paradigm in CLBP_NL comparison > CLBP_L (anterior cingulate and upper and middle frontal gyrus)

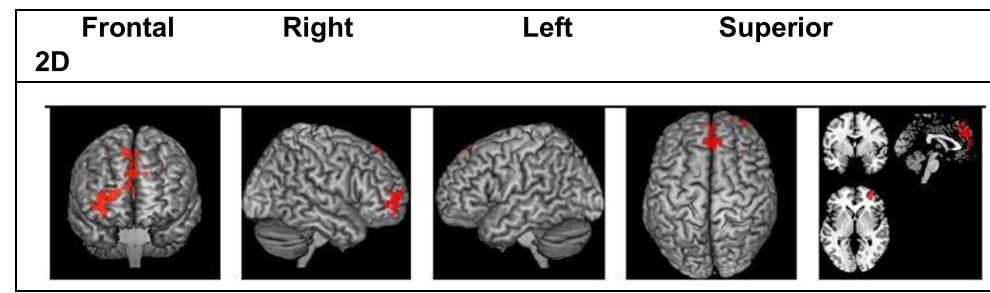

BOLD Effect, values referring to the group average

\begin{tabular}{|c|c|l|l|}
\hline Variable & CLBP_NL & \multicolumn{1}{|c|}{ CLBP_L } & Valor $p$ \\
\hline BOLD & 0,10 & $-0,09$ & \\
Effect & {$[0,04 ; 0,22]$} & {$[-0,23 ;-$} & $<0,001$ \\
& & $0,02]$ & \\
\hline
\end{tabular}

Measurements expressed in Median [Interquartile Interval]. $P$ value: comparison between CLBP_NL and CLBP_L. To compare them as the groups were used MannWhitney and Kruskal-Wallis tests with corrected post-tests by the Holm method.
Graphic boxplot of the BOLD effect, distribution of anterior cingulate and frontal gyrus between groups

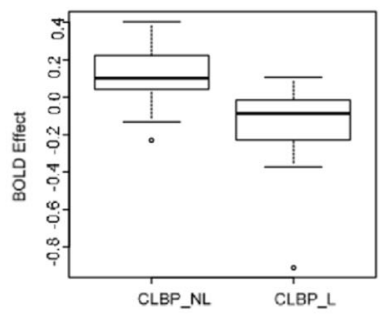

Result of fMRI analysis in pain paradigm in CLBP_L comparison > control (Right Frontal pole and paracingulate region)

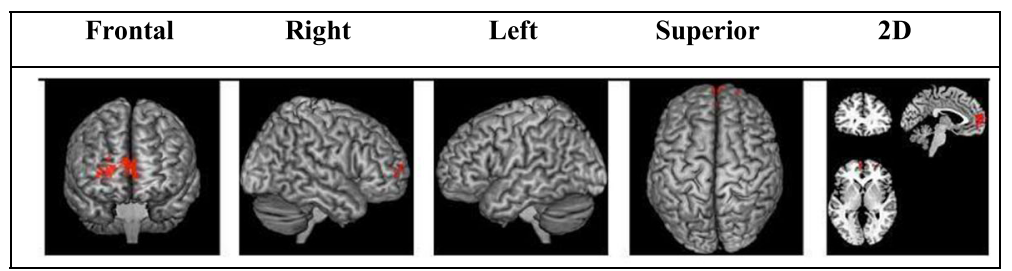

BOLD Effect, values referring to the group average

\begin{tabular}{|c|c|c|c|}
\hline Variable & Control & CLBP_L & Valor $p$ \\
\hline BOLD & 0,07 & $-0,17$ & \\
Effect & {$[-0,04 ; 0,19]$} & {$[-0,37 ;-0,10]$} & $<0,001$ \\
\hline
\end{tabular}

Measurements expressed in Median [Interquartile Interval]. $\mathrm{P}$ value: comparison between control and CLBP_L. To compare them as the groups were used MannWhitney and Kruskal-Wallis tests with corrected post-tests by the Holm method
Graphic boxplot of the BOLD effect, distribution of right frontal pole and paracingulate region between groups

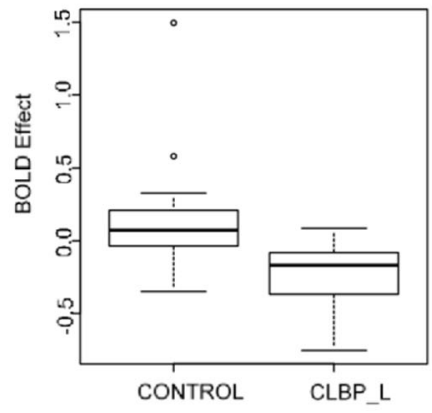

Fig. 2 3D image of brain containing result of fMRI covariate analysis considering the weight used in the pain paradigm

sick-leave demands and associated emotional and economic burden. Low back pain is the leading cause of years lived with disability in both developed and developing countries with profound economic effect [28]. Three decades ago Waddell noted the diminishing probability of returning to work with increasing time away from work [8]. The objective of this study was to compare the neuronal correlates of CLBP individuals with or without sick-leave demands. To the best of our knowledge this is the first study comparing functional neuroimaging in these two groups.

Individuals in our study practiced professions commonly associated with back pain and sick-leave demands. The group CLBP_L had a lower education level, more anxiety, depression, fear avoidance and disability.

Studies have shown an association between painrelated fear of movement as measured by the FearAvoidance Beliefs Questionnaire (FABQ) and treatment response [29]. A positive correlation has been reported between FABQ scores and disability, pain, and reduced return-to-work rate. It has been suggested that fear avoidance beliefs could increase pain perception [30]. In the same manner depression has been linked to increased risk for chronicity in nonspecific back pain, although this relationship is considered bi-directional [31]. 
In our study, we performed an analysis covariating with the weight set individually to match the perceived pressure used in the fMRI acute stimulus. Giesecke et al. [17], reported that individuals with CLBP and control group experienced the same pain intensity when different levels of pressure were applied. In this analysis covariating by objective weight, we verified an increase in the fMRI signal at the anterior cingulate and right superior/ middle frontal gyrus in the CLBP_NL group compared with CLBP_L individuals. We also noted an increase in fMRI signal in the control (asymptomatic) group at the frontal pole and paracingulate cortex in comparison with the CBLP_L group.

The superior frontal gyrus (GFS) includes the premotor cortex and supplementary motor, dorsolateral prefrontal cortex (associated with logic and calculations) and prefrontal cortex (related to planning, decision-making thoughts, attention and working memory) [32]. The activity of the prefrontal cortex medial (mCPF) is correlated with the amount of pain reported by patients and with spontaneous pain. Increased frontal activity may be due to improved cognitive and emotional processing ${ }^{13}$.

Brain activity related to the subjective perception of chronic pain may be distinct from brain activity related to acute pain [33] evaluated fMRI brain activity patterns in the transition from acute to chronic pain. They showed that brain activity in the subacute phase was limited to regions normally involved in acute pain whereas in the chronic back pain group, activity was confined to emotion-related circuitry.

In our study, we did not evaluate spontaneous back pain, but rather pressureevoked acute pain applied at a neutral site. Baliki, et al., [34] studied chronic back individuals while rating their spontaneous pain and compared back pain individuals with a control asymptomatic group for pain response to thermal stimulation. The authors described a double-dissociation between the medial prefrontal cortex (mPFC) activity and insula. While brain activity in the mPFC correlated with the intensity of spontaneous pain, brain regions commonly observed for acute pain correlated with thermal stimulation (the mPFC is known to be involved in negative emotions especially in relation to the self).

A recent systematic review analyzed the available literature on structural and functional brain abnormalities in chronic low back pain. Six studies evaluated brain activity in response to a nociceptive stimulus, three of these with mechanical stimulation.

Overall these studies disclosed some evidence of increased activity in pain-related regions and decreased activity in analgesic regions [35].

Our study also showed an increased activity in primary sensory regions related to pain, but without a significant difference between the groups. However, when we considered the difference in objective pressured applied and perceived pain, we did find a reduced fMRI signal in a frontal region usually related to the processing and emotional aspects of pain, in the CBLP_L group when compared to the CLBP_NL and control group. We hypothesize pos hoc that these areas could be already chronically activated in individuals on sick-leave demands. Negative emotions are associated with increased activation in the anterior cingulate córtex [30] and other areas that mediate the processing of emotions and are important nodes that tend to toward pain.

Individuals with CLBP (or other chronic pain conditions) are more sensitive and activate to greater number of cortical areas related to the pain matrix when compared to individuals without pain [12]. The idea that individuals with CLBP are thought to be "more sensitive" to pain is interesting mainly when clinically evaluating this patient, that frequently presenting with medically unexplained persistent of pain.

Further studies are needed to verify whether individuals on sick-leave demands can be regarded as a distinct subgroup with specific risk factors and distinctive physiopathology mechanisms for chronicity, thus requiring differential treatment from that usually used for the broad-spectrum of CLBP patients.

\section{Conclusion}

The modulation of acute pain participates in the mechanism propagating chronic pain perception. The lower activation in the superior frontal gyrus observed in the CLBP_L group compared to CLBP_NL, reinforces the idea of an already existing activation in this area.

\footnotetext{
Acknowledgements

We would like to thank the Brain Institute of Hospital Israelita Albert Einstein and Rheumatology Division, HCFMUSP Clinical Hospital, Faculty of Medicine, University of Sao Paulo for all the support in conducting this clinical research.
}

\section{Disclosures}

The authors declare no conflict of interest.

\section{Authors' contributions}

AF: Participated in the collection and data discussion and the article writing. LSG: Participated in the study data collection, statistical analysis and the article writing. EAJ, ASRH: Participated in the elaboration of the research, data discussion and the article writing. EFB: Participated in the data discussion and the article writing. LLG: Participated in the collection data. The authors read and approved the final manuscript.

\section{Funding}

This work was supported by the IIEP: Instituto Israelita de Ensino e Pesquisa Albert Einstein - São Paulo, SP, Brazil (CEP/Einstein n¹0/1480) and Coordenação de Aperfeiçoamento de Pessoal de Nível Superior do Ministerio de Educação (CAPPesq 0186/11). Supported by grants from Conselho Nacional de Desenvolvimento Científico e Tecnológico (CNPq \#306879/2018-2 to EFB) and CAPES \#88882.328107/2014-01 for AF).

Availability of data and materials

Materials and data available for consultation. 


\section{Ethics approval and consent to participate}

The study was approved by the Ethics Committee of both institutions (CAPPesq 0186/11 e CEP/Einstein n010/1480).

All individuals signed the informed consent form.

\section{Consent for publication}

Not applicable.

\section{Competing interests}

The authors declare that they have no competing interests.

Received: 21 December 2019 Accepted: 14 August 2020

Published online: 01 September 2020

\section{References}

1. Manchikanti L, Singh V, Falco FJ, Benyamin RM, Hirsch JA. Epidemiology of low back pain in adults. Neuromodulation. 2014;17 Suppl 2:3-10. https://doi. org/10.1111/ner.12018

2. Meucci RD, Fassa AG, Faria NMX. Prevalence of chronic low back pain: systematic review. Rev Saúde Pública [Internet]. 2015:49-73[cited 2020 Mar 15]. Available from: Epub Oct 20, 2015. https://doi.org/10.1590/S0034-8910. 2015049005874.

3. Fernandes JA. Initial approach to patients with acute lower back pain. Rev Assoc Med Bras. 2016;62(2):186-91. https://doi.org/10.1590/1806-9282.62.02. 188 [cited 2020 Mar 15].

4. Shmagel A, Foley R, Ibrahim H. Epidemiology of chronic low Back pain in US adults: data from the 2009-2010 National Health and nutrition examination survey. Arthritis Care Res (Hoboken). 2016;68(11):1688-94. https://doi.org/10.1002/acr.22890.

5. Wertli MM, Rasmussen-Barr E, Weiser S, Bachmann LM, Brunner F. The role of fear avoidance beliefs as a prognostic factor for outcome in patients with nonspecific low back pain: a systematic review. Spine J. 2014;14(5):816-36. e4. https://doi.org/10.1016/.jspinee.2013.09.036

6. Mordeniz C. Pain perception within consciousness. NeuroQuantology. 2016: 14. https://doi.org/10.14704/ng.2016.14.2.957.

7. Cancelliere C, Donovan J, Stochkendahl MJ, Biscardi M, Ammendolia C, Myburgh C, et al. Factors affecting return to work after injury or illness: best evidence synthesis of systematic reviews. Chiropr Man Therap. 2016;24(1):32. https://doi.org/10.1186/s12998-016-0113-z.

8. Waddel G. A new clinical model for the treatment of low Back pain. Spine. 1987;12(7):632-44

9. Meziat NF, Silva GA. Disability pension from back pain among social security beneficiaries, Brazil. Rev Saúde Pública. 2011;45(3):494-502.

10. Meucci RD, Fassa AG, Paniz VM, Silva MC, Wegman DH. Increase of chronic low back pain prevalence in a medium-sized city of southern Brazil. BMC Musculoskelet Disord. 2013;14:155. https://doi.org/10.1186/1471-2474-14-155.

11. Morton DL, Sandhu JS, Jones AK. Brain imaging of pain: state of the art. J Pain Res. 2016;9:613-24. https://doi.org/10.2147/JPR.S60433.

12. Apkarian AV. The brain in chronic pain: clinical implications. Pain Management. 2011;1 (6):577-86

13. Amaro E Jr, Barker GJ. Study design in fMRI: basic principles. Brain Cogn. 2006;60(3):220-32.

14. Ogawa S, Lee TM, Kay AR, Tank DW. Brain magnetic resonance imaging with contrast dependent on blood oxygenation. Proc Natl Acad Sci U S A. 1990;87(24):9868-72.

15. Geha P, Waxman SG. Pain perception: multiple matrices or one? JAMA Neurol. 2016. https://doi.org/10.1001/jamaneurol.2016.0757.

16. Vachon-Presseau E, Centeno MV, Ren W, Berger SE, Tétreault P, et al. The emotional brain as a predictor and amplifier of chronic pain. J Dent Res. 2016;95(6):605-12. https://doi.org/10.1177/0022034516638027.

17. Giesecke T, Gracely RH, Grant MA, Nachemson A, Petzke F, Williams DA, et al. Evidence of augmented central pain processing in idiopathic chronic low back pain. Arthritis Rheum. 2004;50(2):613-23.

18. Davis KD. Neuroimaging of pain: what does it tell us? Curr Opin Support Palliart Care. 2011;5(2):116-21. https://doi.org/10.1097/SPC. 0b013e3283458f96.

19. Nusbaum L, Natour J, Ferraz MB, et al. Translation, adaptation and validation of the Roland-Morris questionnaire -- Brazil Roland-Morris. Braz J Med Biol Res. 2001;34:203-10.
20. Gracely RH, Geisser ME, Giesecke T, Grant MA, Petzke F, Williams DA, et al. Pain catastrophizing and neural responses to pain among persons with fibromyalgia. Brain. 2004;127(Pt 4):835-43.

21. Beck AT, Ward CH, Mendelson M, Mock J, Erbaugh J. An inventory for measuring depression. Arch Gen Psychiatry. 1961;4:561-71 PubMed PMID: 13688369.

22. Fioravanti ACM, Santos LF, Maissonette S, Cruz APM, Landeira-Fernandez J. Avaliação da estrutura fatorial da Escala de Ansiedade-Traço do IDATE. Aval psicol. 2006;5(2):217-24 [citado 2019 Dez 20].

23. Abreu AM, Faria CDCM, Cardoso SMV, Teixeira-Salmela LF. Versão Brasileira do fear avoidance beliefs questionnaire. Cad Saúde Pública. 2008;24(3):61523.

24. Woolrich MW, Ripley BD, Brady M, Smith SM. Temporal autocorrelation in univariate linear modeling of FMRI data. Neuroimage. 2001;14(6):1370-86.

25. Faraway JJ. Extending the linear model with $\mathrm{R}$ : generalized linear, mixed effects and nonparametric regression models. Boca Raton: Chapman \& Hall/ CRC; 2006.

26. SPSS Inc. Released. SPSS statistics for windows, version 17.0. Chicago: SPSS Inc.. Released; 2008.

27. R Core Team. R: a language and environment for statistical computing. Vienna: R Foundation for statistical computing; 2015. URL http://www.Rproject.org/.

28. Maher C, Underwood M, Buchbinder R. Non-specific low back pain. Lancet. 2017:389:736-47. https://doi.org/10.1016/s0140-6736(16)30970-9.

29. Feitosa AS, Lopes JB, Bonfa E, Halpern AS. A prospective study predicting the outcome of chronic low back pain and physical therapy: the role of fear-avoidance beliefs and extraspinal pain. Rev Bras Reumatol. 2016. https://doi.org/10.1016/j.rbr.2015.11.001.

30. Garland EL. Pain processing in the human nervous system: a selective review of nociceptive and biobehavioral pathways. Prim Care. 2012;39(3): 561-71. https://doi.org/10.1016/j.pop.2012.06.013.

31. Fernandez M, Colodro-Conde L, Hartvigsen J, Ferreira ML, Refshauge KM, Pinheiro MB, et al. Chronic low back pain and the risk of depression or anxiety symptoms: insights from a longitudinal twin study. Spine J. 2017; 17(7):905-12. https://doi.org/10.1016/j.spinee.2017.02.009.

32. Vernet $M$, Quentin $R$, Chanes $L$, Mitsumasu A, Valero-Cabré A. Frontal eye field, where art thou? Anatomy, function, and non-invasive manipulation of frontal regions involved in eye movements and associated cognitive operations. Front Integr Neurosci. 2014;8:66Published 2014 Aug 22. https:// doi.org/10.3389/fnint.2014.00066

33. Hashmi JA, Baliki MN, Huang L, Baria AT, Torbey S, Hermann KM, et al. Shape shifting pain: chronification of back pain shifts brain representation from nociceptive to emotional circuits. Brain. 2013;136(9):2751-68. https:// doi.org/10.1093/brain/awt211.

34. Baliki MN, Chialvo DR, Geha PY, Levy RM, Harden RN, Parrish TB, et al. Chronic pain and the emotional brain: specific brain activity associated with spontaneous fluctuations of intensity of chronic back pain. J Neurosci. 2006; 26(47):12165-73.

35. Kregel J, Meeus M, Malfliet A, Dolphens M, Danneels L, Nijs J, et al. Structural and functional brain abnormalities in chronic low back pain: a systematic review. Semin Arthritis Rheum. 2015;45(2):229-37.

\section{Publisher's Note}

Springer Nature remains neutral with regard to jurisdictional claims in published maps and institutional affiliations.

Ready to submit your research? Choose BMC and benefit from:

- fast, convenient online submission

- thorough peer review by experienced researchers in your field

- rapid publication on acceptance

- support for research data, including large and complex data types

- gold Open Access which fosters wider collaboration and increased citations

- maximum visibility for your research: over $100 \mathrm{M}$ website views per year

At $\mathrm{BMC}$, research is always in progress.

Learn more biomedcentral.com/submissions 